
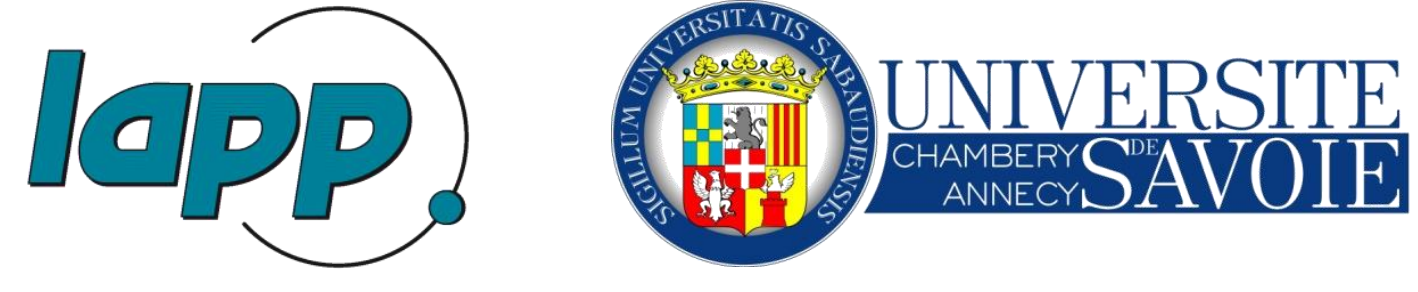

\title{
The ATLAS Liquid Argon Calorimeter at the LHC : Overview and Performance
}

Mathieu Aurousseau (LAPP)

On behalf of the ATLAS Liquid Argon Calorimeter group
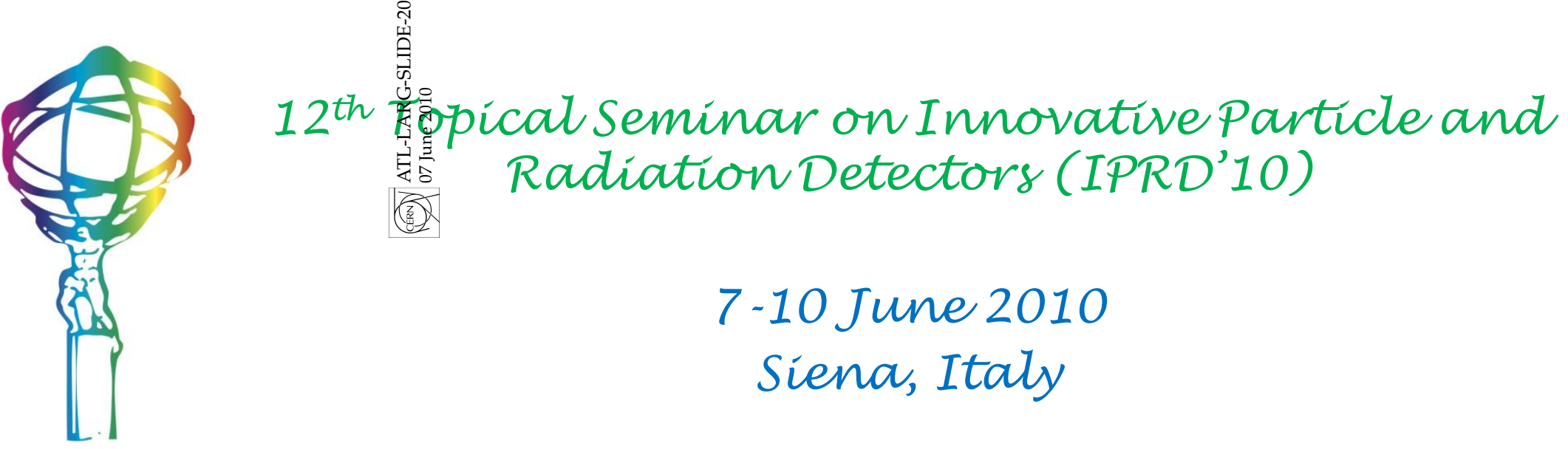

$$
\begin{aligned}
& \text { 7-10 June } 2010 \\
& \text { Siena, Italy }
\end{aligned}
$$




\section{I - Overview of the LAr calorimeters}

- The ATLAS Liquid Argon Calorimeters

- Measurement of Energy

\section{II - Performance of the LAr Calorimeters}

- Stability of calibration constants

- Timing alignment

- Calorimeter trigger

- Uniformity and energy resolution with cosmics and $\pi^{0}$

- Energy flow with LHC collisions

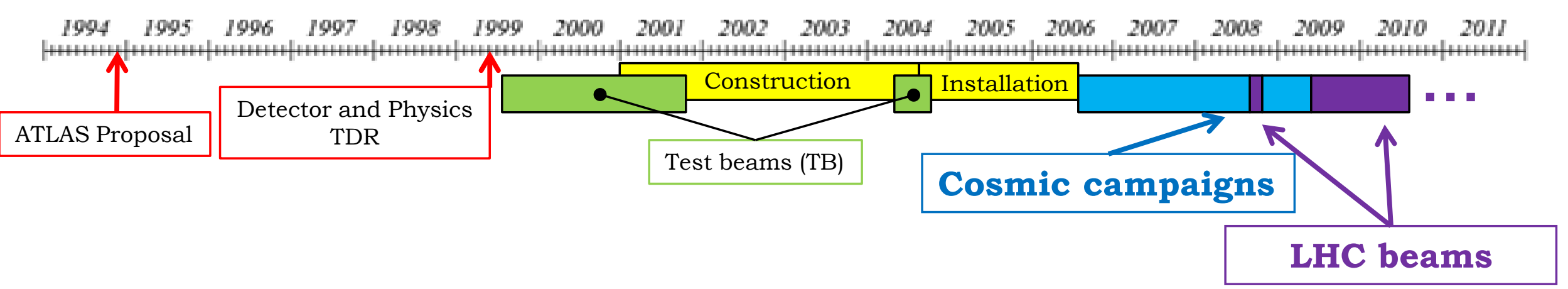




\section{I- Overview of the LAr Calorimeters}









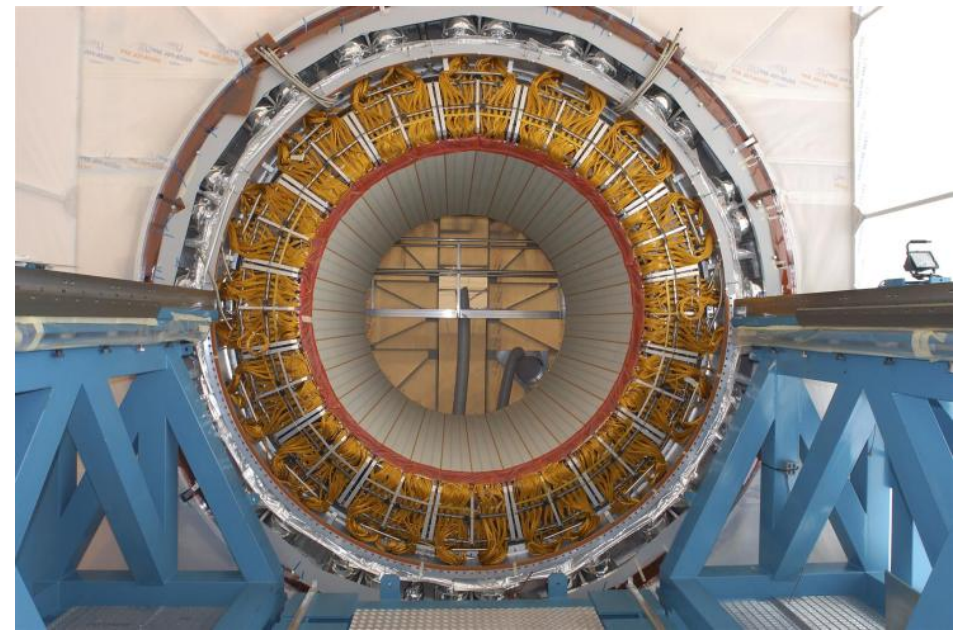

\section{- EM calorimeter}

$\rightarrow \mathrm{Pb}+\mathrm{LAr}$

$\rightarrow$ Accordion geometry

- full $\phi$ coverage without crack

$\rightarrow$ Coverage $:|\eta|<3.2$

$\rightarrow$ Design resolution (measured in TB)

$$
\frac{\Delta E}{E}=\frac{10 \%}{\sqrt{E(G e V)}} \oplus 0.7 \%
$$

$\rightarrow 3$ layers up to $|\eta|=2.5 ; 2$ up to $|\eta|=3.2$

$\rightarrow$ Preshower detector up to $|\eta|=1.8$

$\rightarrow 173312$ readout channels $(98.5 \%$ operational)
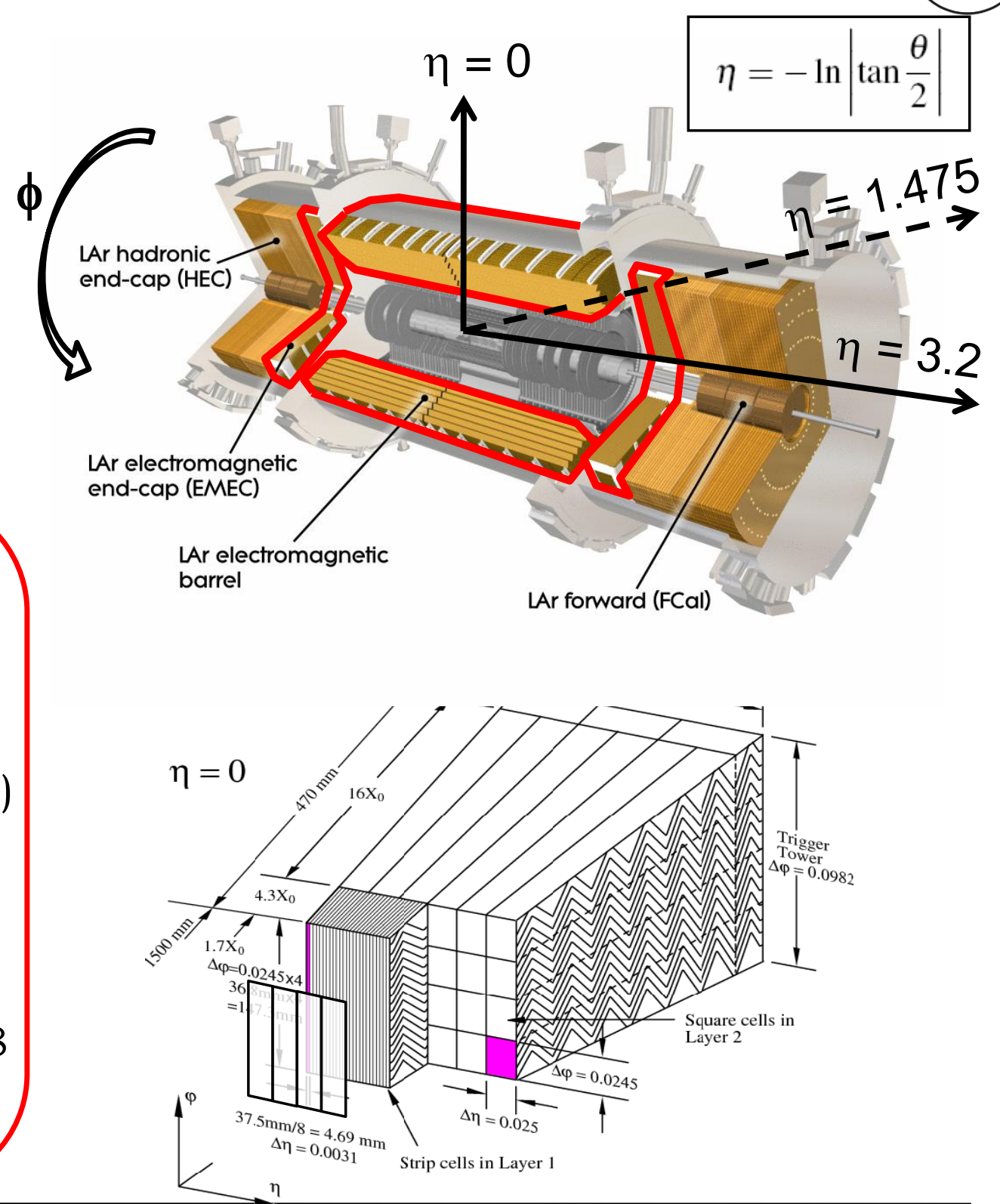


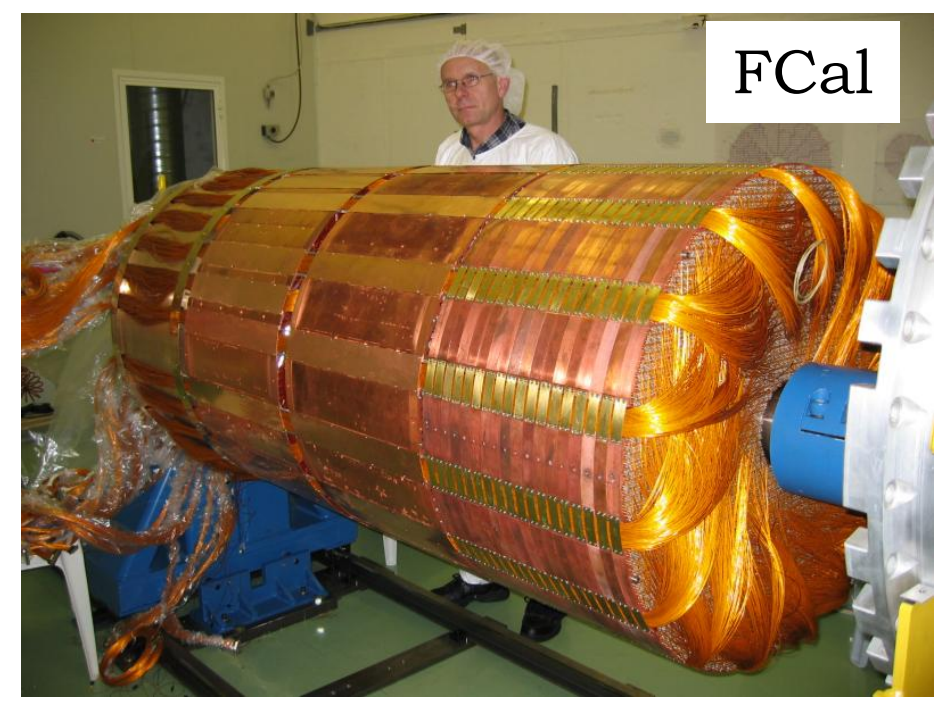

- Forward calorimeter (FCal)

$\rightarrow \mathrm{Cu} / \mathrm{W}$ tubes $+\mathrm{LAr}$

$\rightarrow$ Coverage : $3.1<|\eta|<4.9$

$\rightarrow$ Design resolution (measured in TB)

$$
\frac{\Delta E}{E}=\frac{100 \%}{\sqrt{E(G e V)}} \oplus 10 \%
$$

$\rightarrow 1 \mathrm{EM}, 2$ Hadronic layers

$\rightarrow 3524$ readout channels $(100 \%$ operational)



barrel

- Hadronic Endcap (HEC)

$\rightarrow \mathrm{Cu}+\mathrm{LAr}$

$\rightarrow$ Flat plates

$\rightarrow$ Coverage : $1.5<|\eta|<3.2$

$\rightarrow$ Design resolution (measured in TB)

$\rightarrow 4$ layers

$$
\frac{\Delta E}{E}=\frac{50 \%}{\sqrt{E(G e V)}} \oplus 3 \%
$$

$\rightarrow 5632$ readout channels $(99.9 \%$ operational) 


\section{Measurement of Energy in LAr}

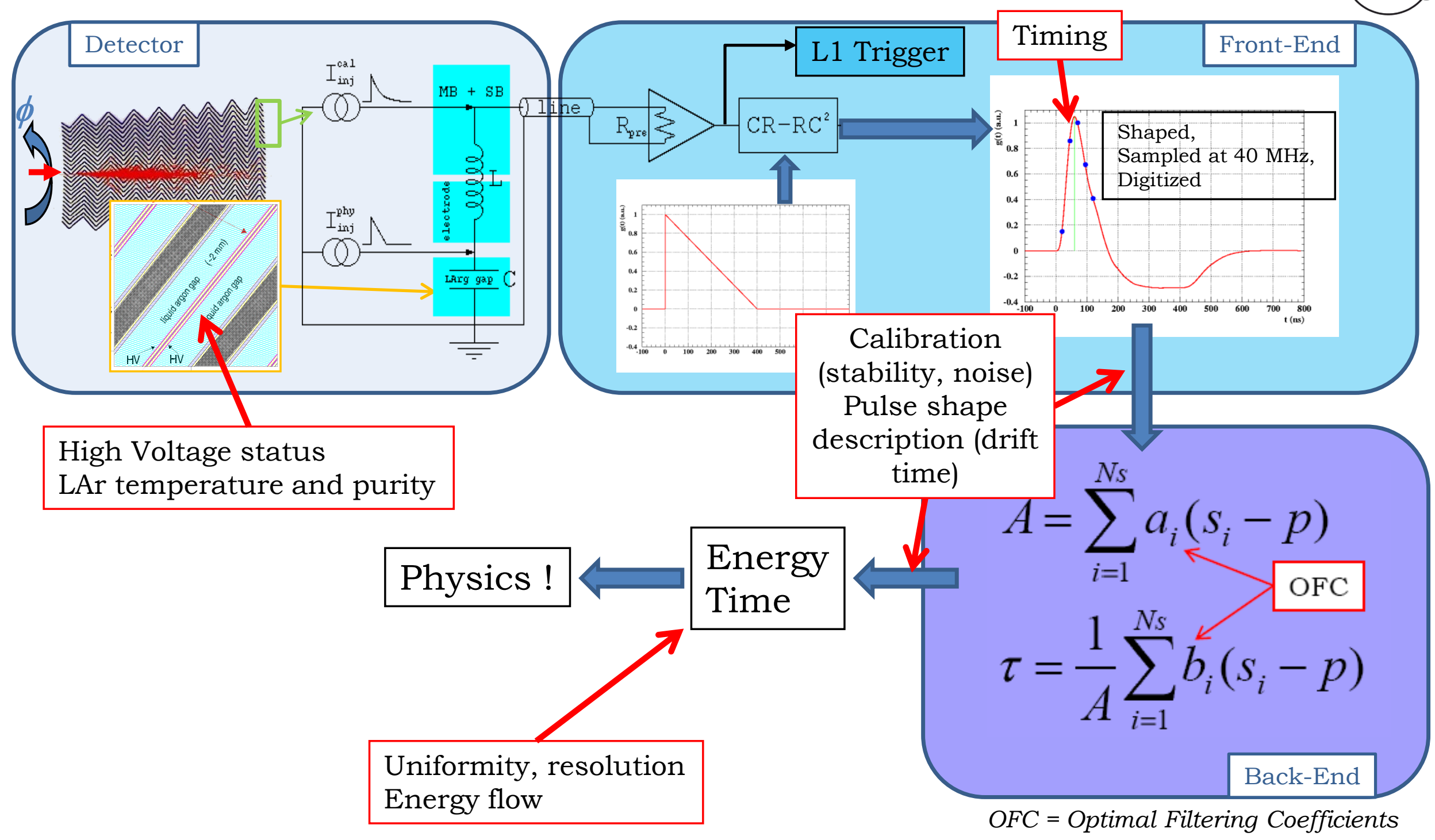




\section{II- Performance of the LAr calorimeters}




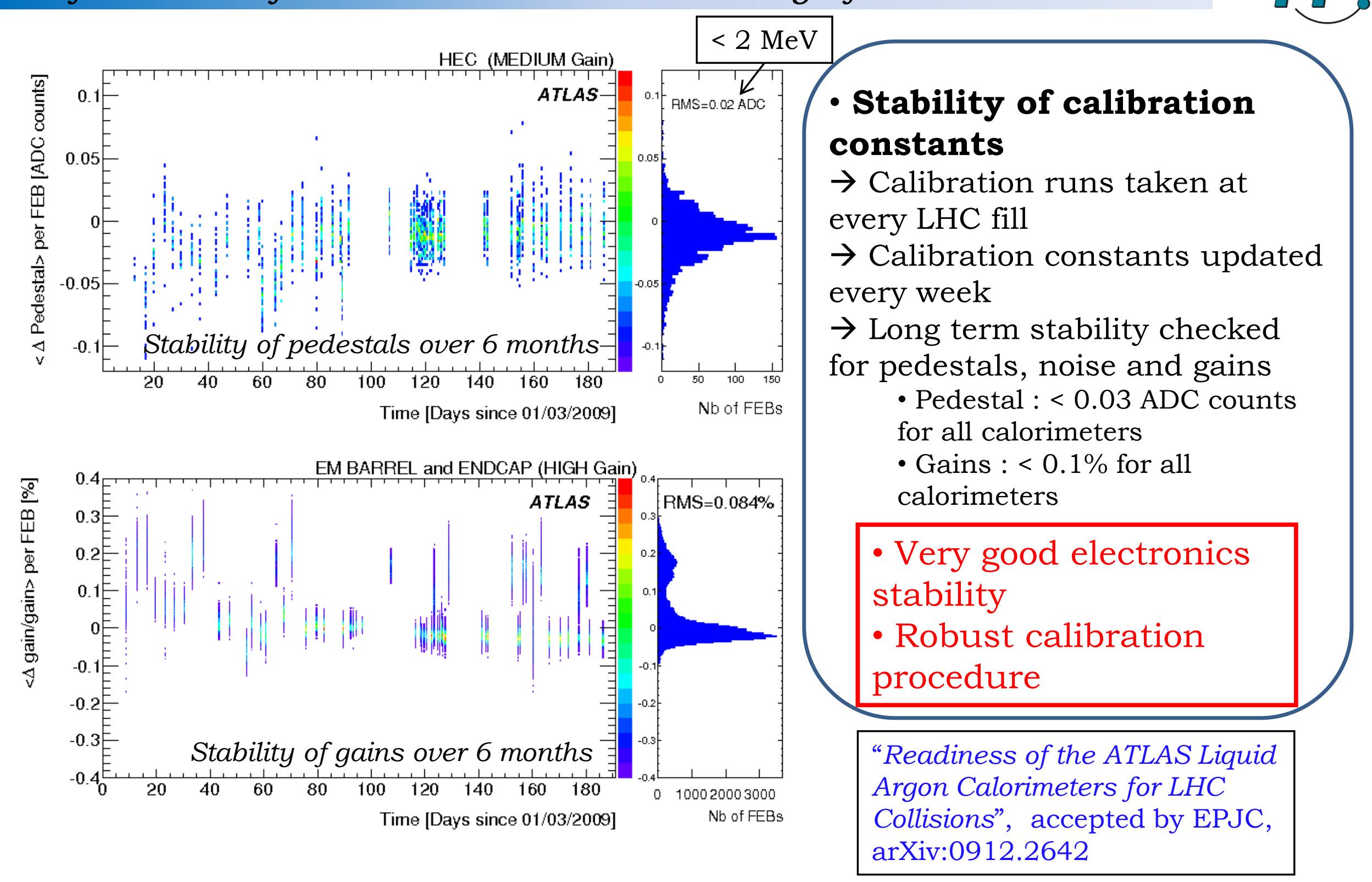




\section{Timing alignment}
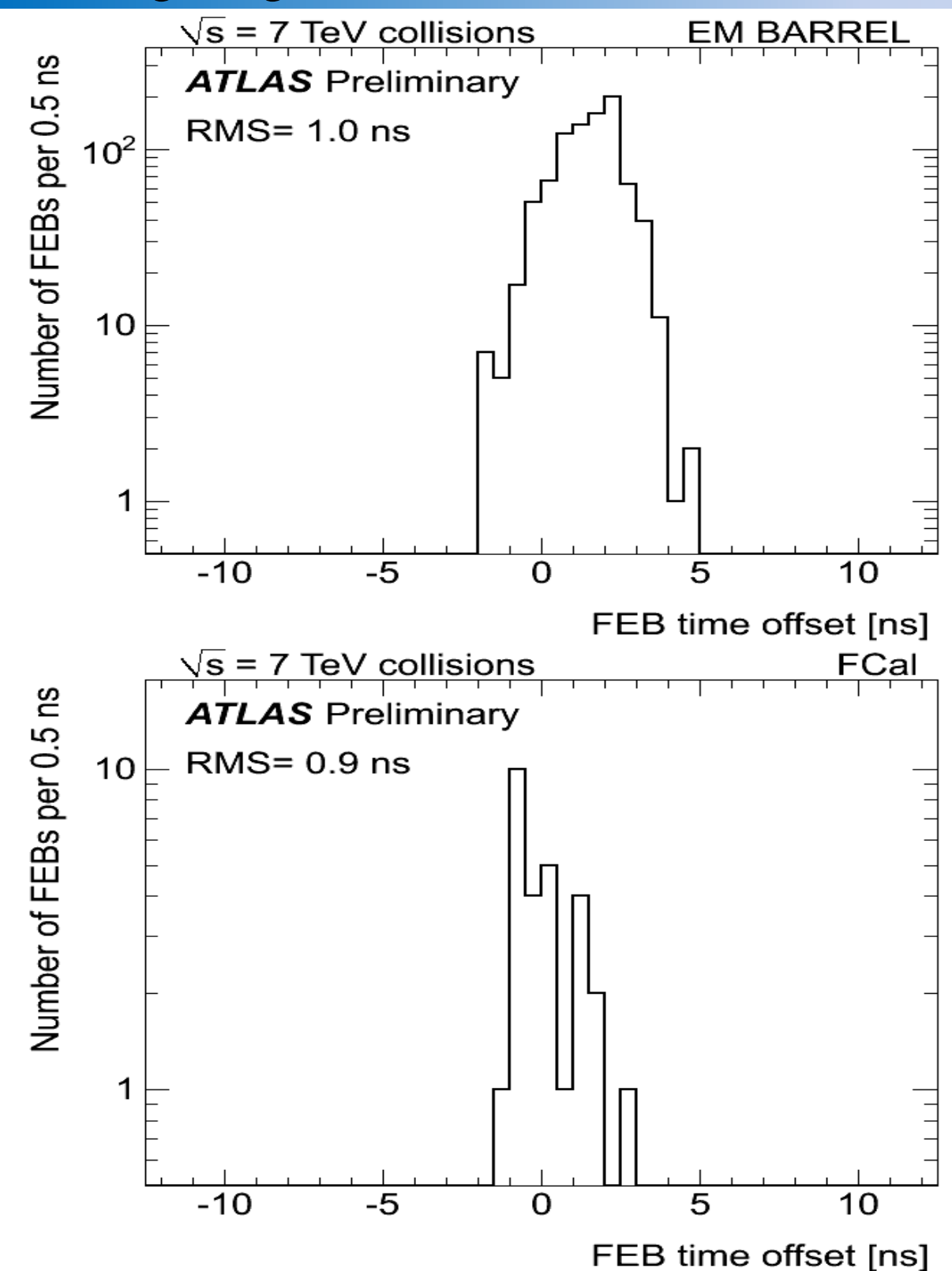

\section{- Timing alignment}

$\rightarrow$ To synchronize readout clock with bunch crossings

$\rightarrow$ Predict delay due to cabling and time of flight

$\rightarrow$ Compute time from data (beamsplashes, cosmics, collisions)

$\rightarrow$ Time offset $=$ difference between measured and predicted delay (averaged over the 128 channels of each Front-End Boards (FEB))

- Aligned at $1 \mathrm{~ns}$, at the FEB level

- Objective : 100ps at the cell level 
Performance of calorimeter trigger with $L H C$ beams
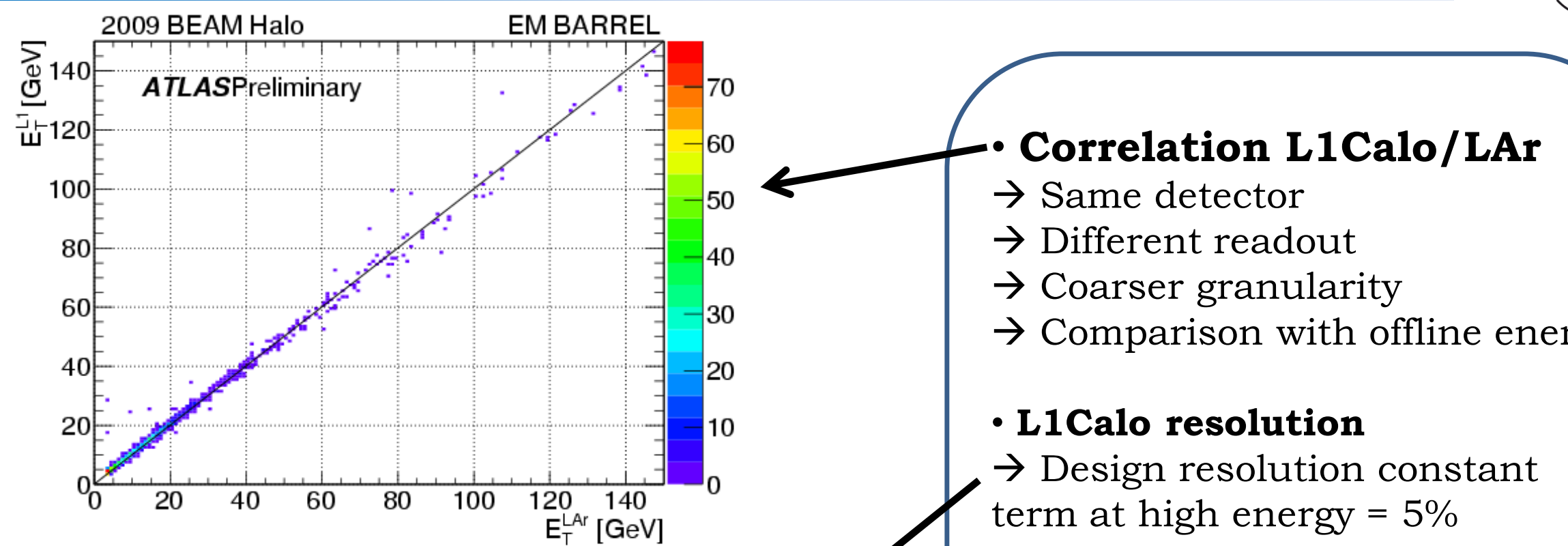

$\rightarrow$ Same detector

$\rightarrow$ Different readout

$\rightarrow$ Coarser granularity

$\rightarrow$ Comparison with offline energy

- L1Calo resolution

$\rightarrow$ Design resolution constant term at high energy $=5 \%$

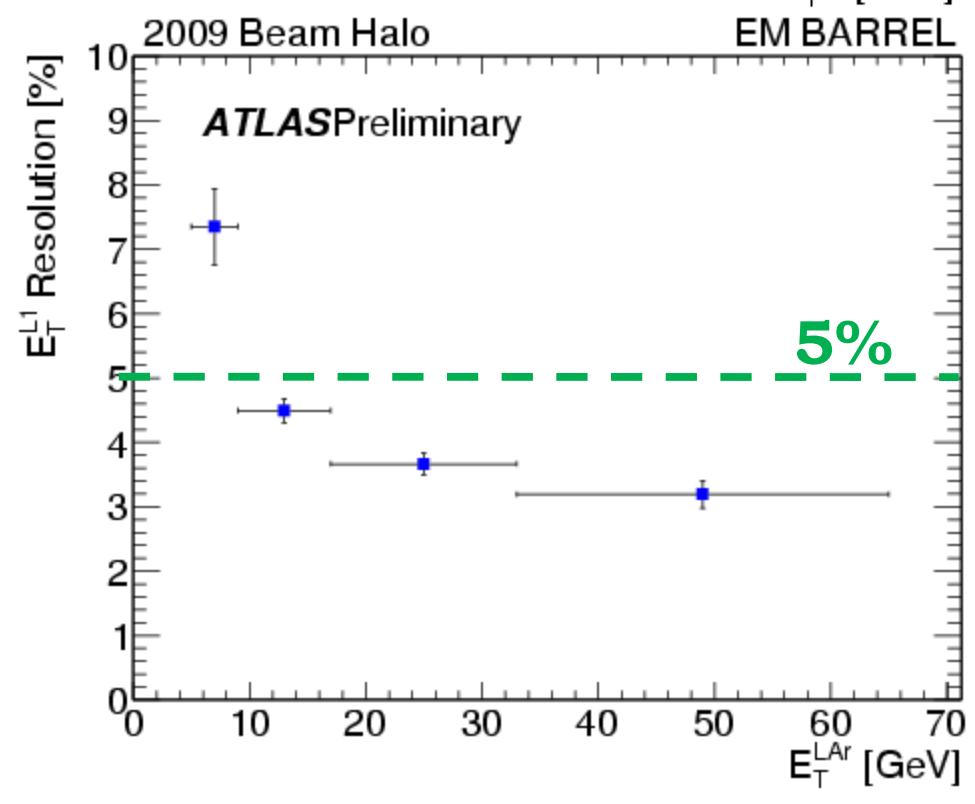

- Good correlation between L1Calo energy and offline LAr energy

- Resolution better than the design constant term 
Uniformity measurements with cosmic muons and $\pi^{0} \rightarrow \gamma \gamma$

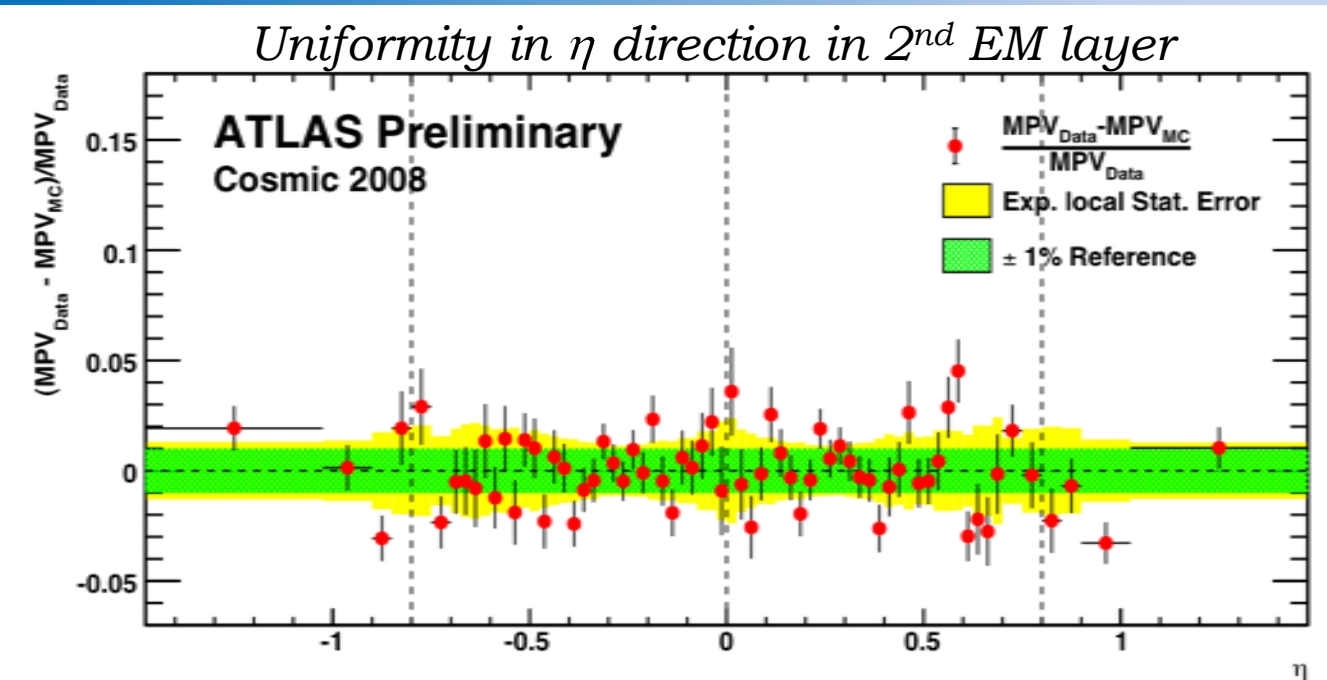

Ratio Data $\pi^{0}$ mass / MC $\pi^{0}$ mass

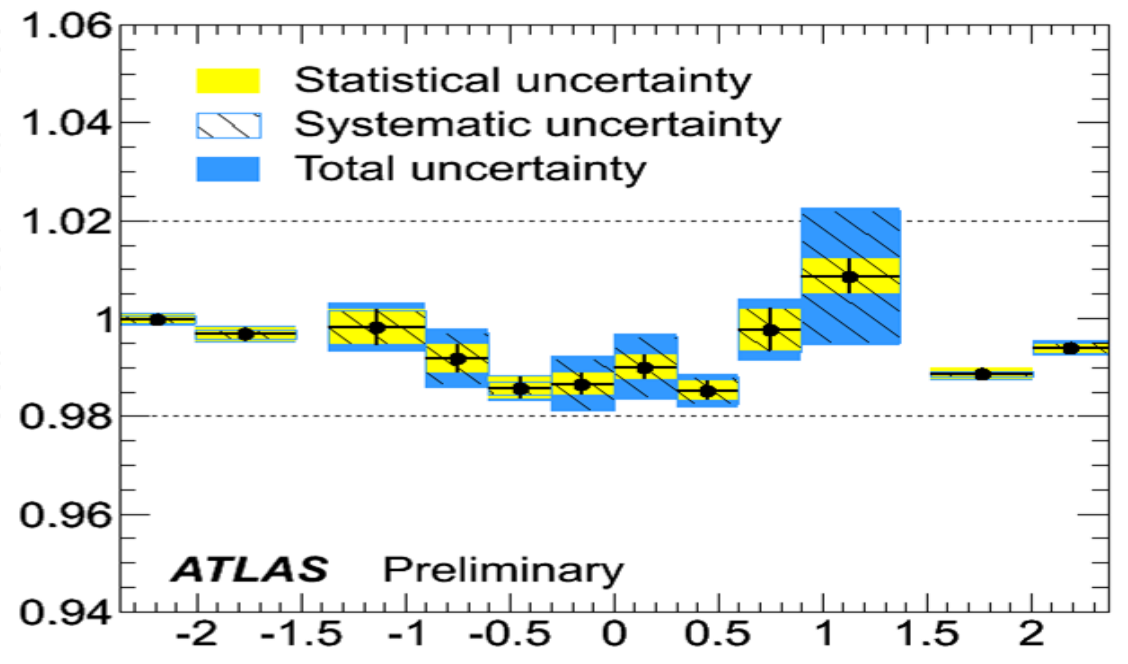

- Uniformity measurement

$\rightarrow$ Linked to resolution constant term

$\rightarrow$ Measured with cosmic muons

- insentitive to material

$\rightarrow$ Measured with $\pi^{0} \rightarrow \gamma \gamma$

- effects of material

Ratio Data $\pi^{0}$ mass / MC $\pi^{0}$ mass

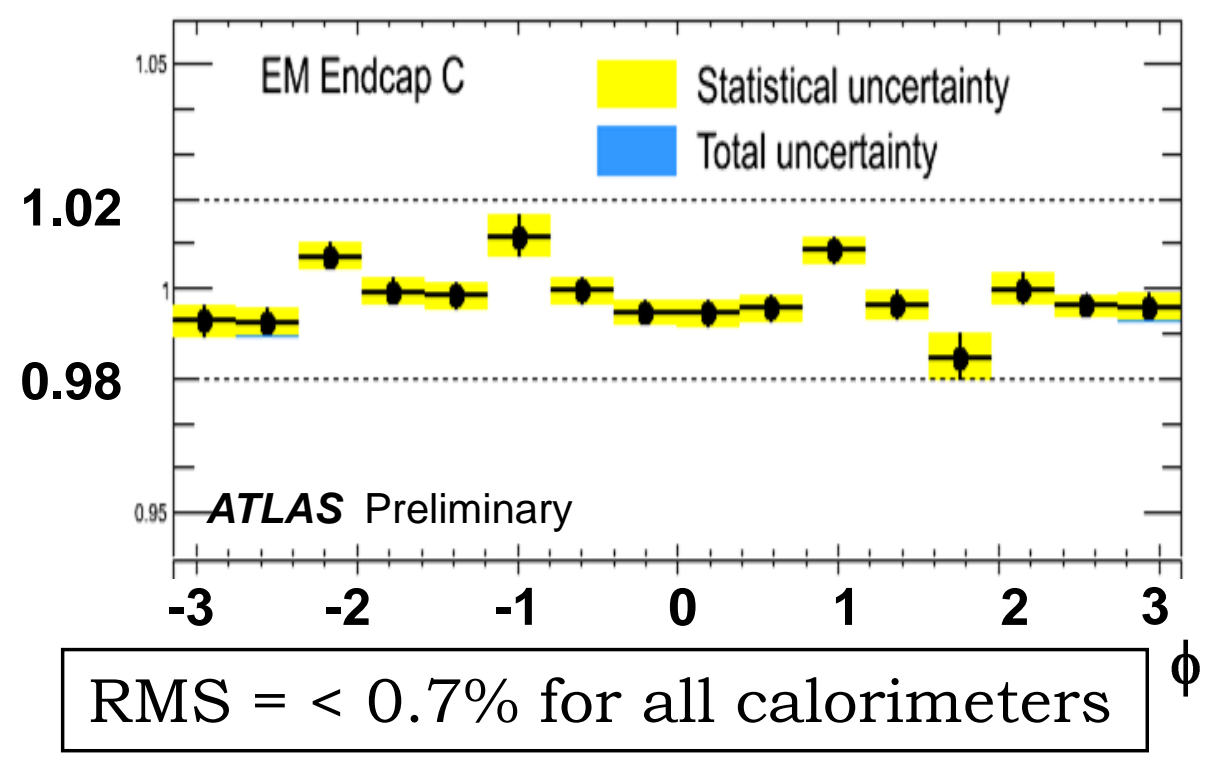




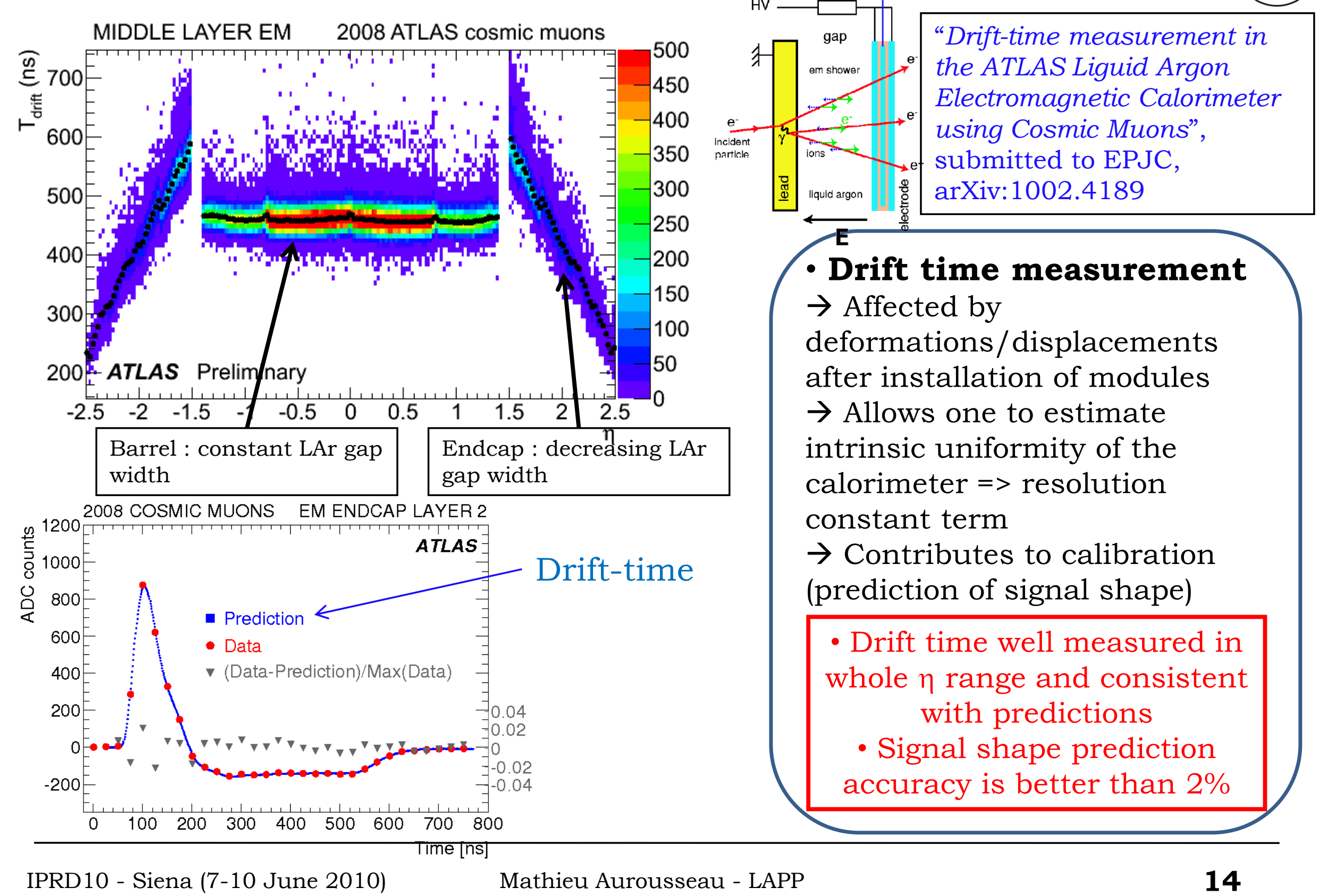




\section{Summary and conclusions}


- LAr calorimeters are in place in the ATLAS cavern since 2006

- Several years of commissioning with test beams, cosmic muons and now LHC collisions

- Performance of LAr tested and well understood

- Control of stability

- Online monitoring of detector status, calibration and data quality well developed (see poster by O. Simard)

- First physics events are seen in the LAr calorimeters

- $\pi^{0}, \mathrm{~J} / \psi$ peak

- W, Z events

- See talk by M. Kuna

Good performance of LAr calorimeters can be expected for the coming physics era! 


\section{Thanks for your attention}




\section{Additional slides}



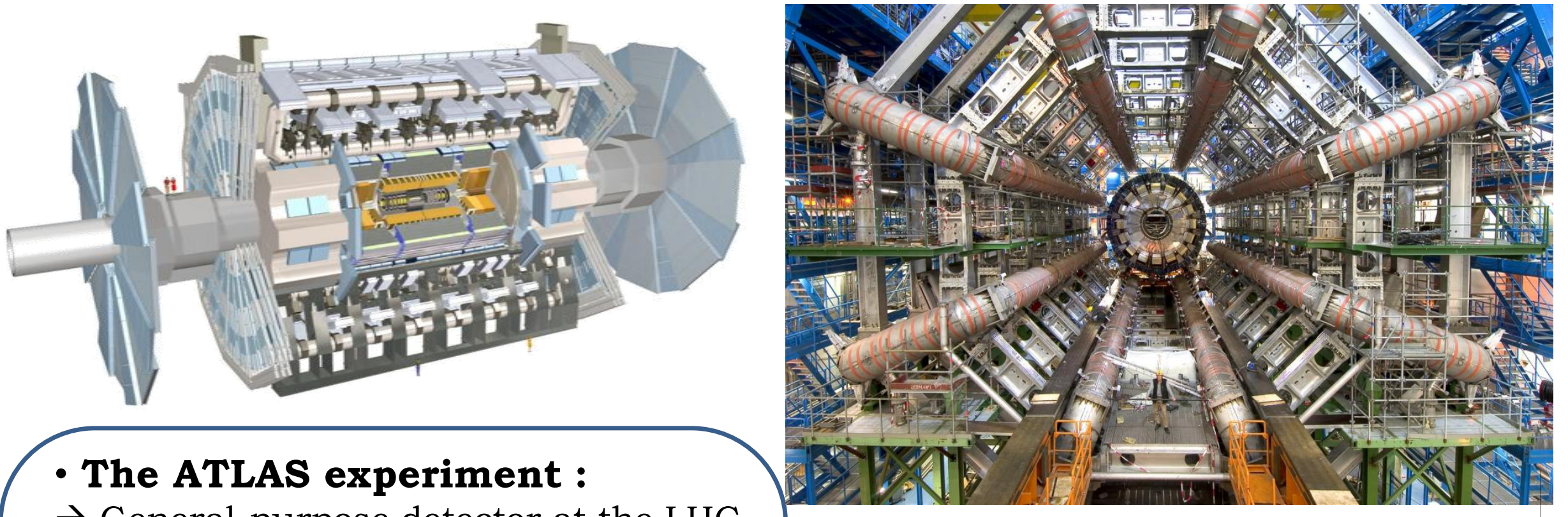

\section{- The ATLAS experiment :}

$\rightarrow$ General-purpose detector at the LHC

$\rightarrow$ Designed to work in the LHC environement :

- high radiation dose

- high interaction rate ( $\mathrm{p}-\mathrm{p}$ collisions every $25 \mathrm{~ns}$ )

\section{- Physics goals :}

$\rightarrow$ Precision Electroweak measurements

$\rightarrow$ Higgs searches

$\rightarrow$ Physics beyond Standard Model

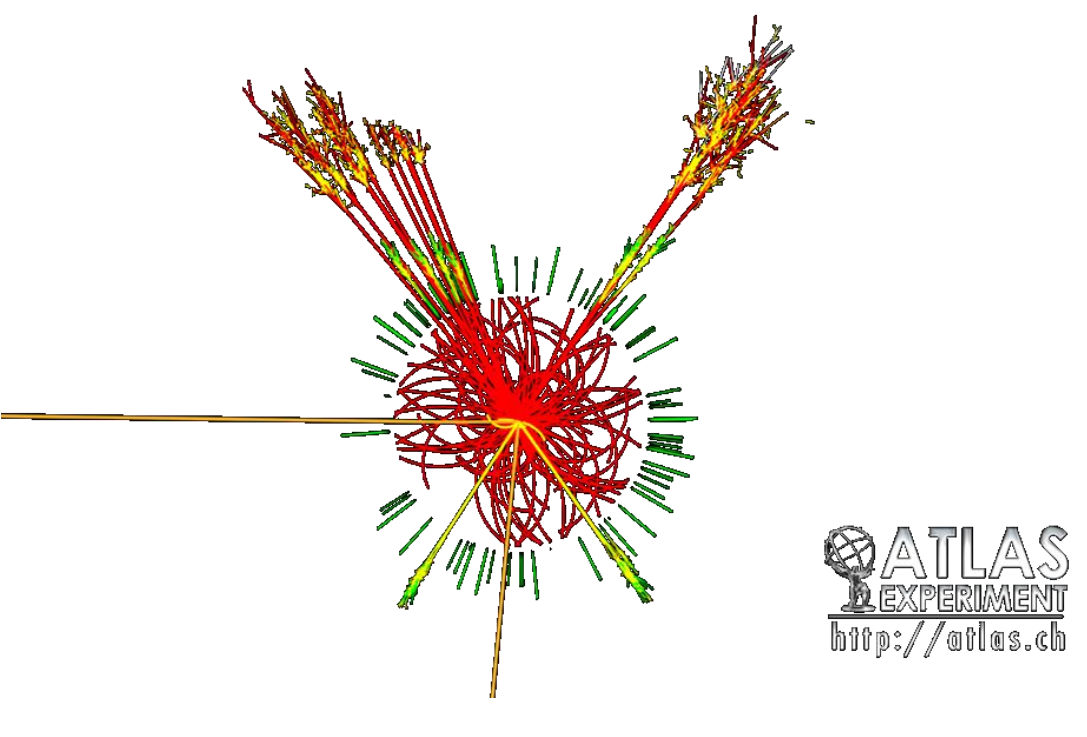




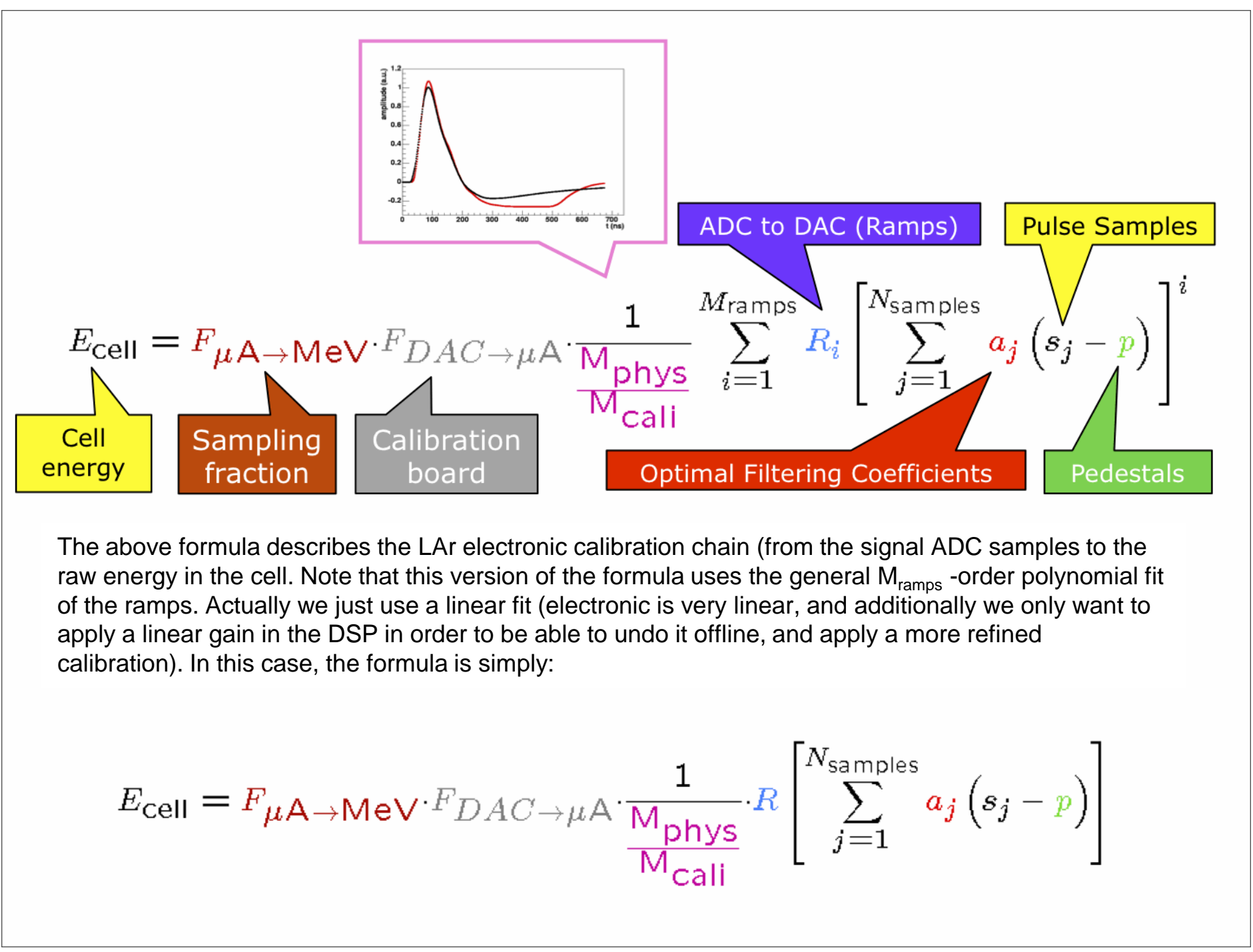




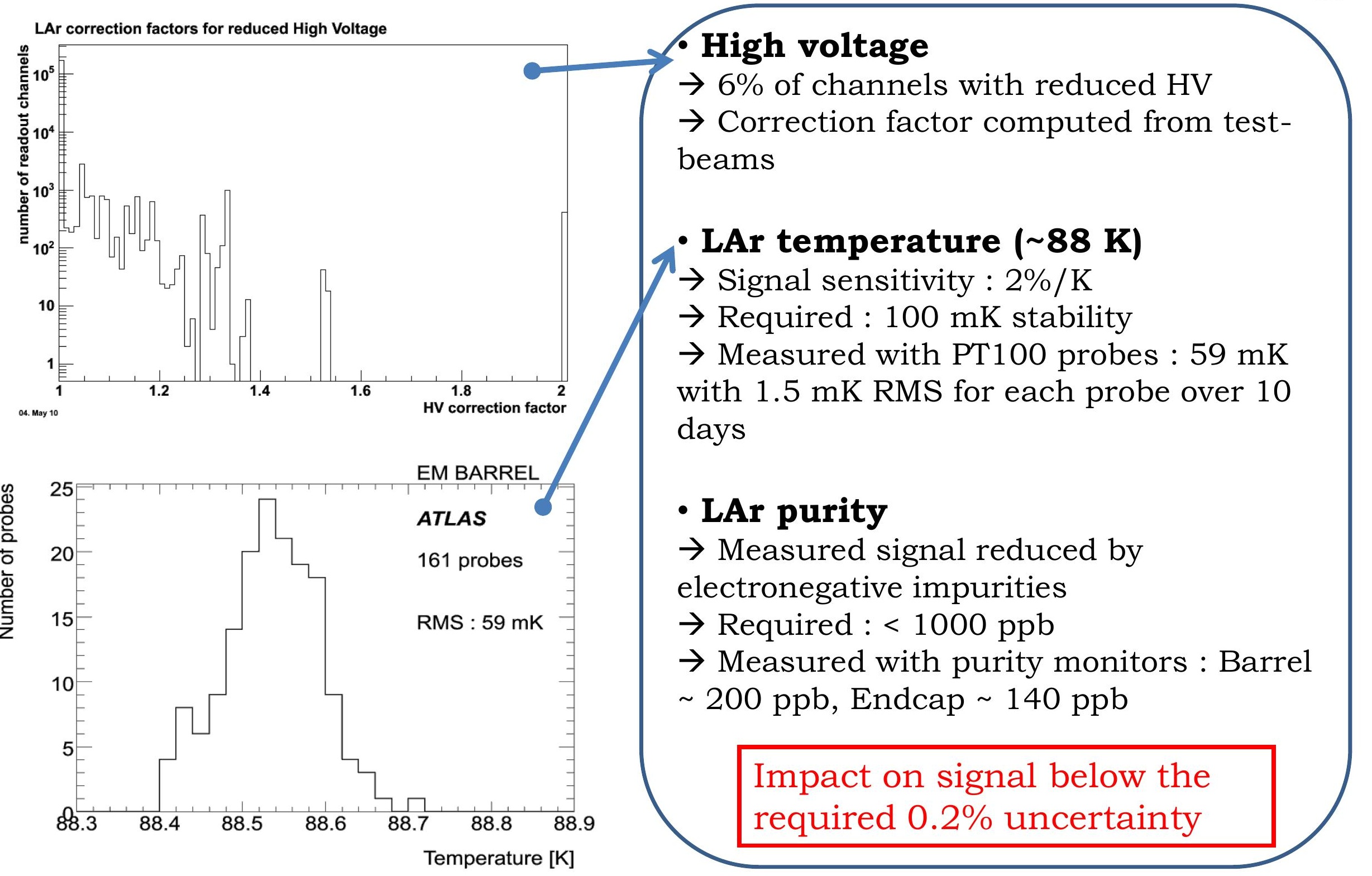

